\title{
Dermoscopic assessment of skin toxicities in patients with melanoma during treatment with vemurafenib
}

\author{
Marcin Rajczykowski ${ }^{1}$, Grazyna Kaminska-Winciorek², Elzbieta Nowara³ ${ }^{3}$ Marzenna Samborska-Plewicka ${ }^{1}$, \\ Sebastian Giebel ${ }^{2}$ \\ ${ }^{1} 2^{\text {nd }}$ Clinic of Radiotherapy and Chemotherapy, Maria Sklodowska-Curie Memorial Cancer Center and Institute of Oncology, \\ Gliwice Branch, Gliwice, Poland \\ ${ }^{2}$ Department of Bone Marrow Transplantation and Onco-Hematology, Maria Sklodowska-Curie Memorial Cancer Center and Institute \\ of Oncology, Gliwice Branch, Poland \\ ${ }^{3}$ Clinical and Experimental Oncology Department, Maria Sklodowska-Curie Memorial Cancer Center and Institute of Oncology, \\ Gliwice Branch, Poland
}

Adv Dermatol Allergol 2018; XXXV (1): 39-46

DOI: https://doi.org/10.5114/ada.2018.73163

\begin{abstract}
Introduction: The use of vemurafenib in melanoma has improved the survival of patients; however, it is associated with skin toxicities.

Aim: To assess skin toxicities by dermoscopy in patients treated with vemurafenib.

Material and methods: Eight patients with BRAF V600 mutation positive metastatic melanoma were examined dermoscopically during vemurafenib treatment. All skin lesions occurring during therapy were assessed clinically and dermoscopically using a hand-held dermoscope with polarised and non-polarised light. Skin lesions suspected for malignancy appearing during therapy were totally surgically excised with consecutive histopathological examination.

Results: All 8 examined patients developed skin toxicity. The majority of patients (7/8) presented G1 skin toxicity according to CTCAE version 4.3. Only 1 of them had G2 skin toxicity. The most common dermoscopy findings in our study were hyperkeratotic verrucas in 5 patients (5/8) with structureless pattern. In some of them we also observed central dots, exophytic proliferation, hairpin vessels and homogeneous haemorrhage. Other findings were hyperkeratosis of the nipples (5/8) with brownish to yellowish, angular clods with a tendency to be more confluent in dermoscopy. Palmar plantar erythrodysaesthesia (3/8) showed dermoscopically a yellowish, homogeneous pattern. Four melanocytic skin lesions in 2 patients were surgically excised due to suspected malignant transformation. In most of them we observed an atypical pigmented network (abrupt cut-off, big holes), atypical globules and a homogeneous blue pattern; however, histopathological diagnosis excluded any malignancy.

Conclusions: Dermoscopy seems to be an easily performed and valuable method for assessment of skin toxicities during oncological therapy, at any time of the treatment.
\end{abstract}

Key words: dermoscopy, vemurafenib, BRAF inhibitor, skin toxicities, nipple hyperkeratosis.

\section{Introduction}

Melanoma develops from melanocytes and occurs predominantly in the skin. Ocular, meningeal and mucosal locations of this neoplasm are significantly less common [1]. Inoperable metastatic melanoma is an indication for systemic therapy [1]. Over the past decade, we have seen significant progress in the treatment of melanoma. Targeted therapies and immunotherapies have become important therapeutic options [1, 2]. One of the important trials was BRIM-3, a global pivotal trial which proved that vemurafenib therapy reduced the risk of death or progression in patients with confirmed BRAF V600 mutation compared to a group of patients treated with dacarbazine [3]. However, patients treated with vemurafenib developed adverse reactions, including skin toxicities such as rash (9\%), squamous cell carcinoma (19\%), keratoac-

Address for correspondence: Grazyna Kaminska-Winciorek MD, PhD, Department of Bone Marrow Transplantation and Onco-Hematology, Maria Sklodowska-Curie Memorial Cancer Center and Institute of Oncology, 15 Wybrzeze Armii Krajowej St, 44-100 Gliwice, Poland, phone: +48 698626 208, fax: +48 3227891 49, e-mail: dermatolog.pl@gmail.com

Received: 23.12.2016, accepted: 15.02.2017. 
anthoma (10\%), and even new primary melanoma (2\%) [3]. According to a recent report on skin toxicities during vemurafenib therapy in a group of 107 patients, the most common clinically significant skin-related adverse events were rash (64\%), squamoproliferative growths (41\%), photosensitivity (40\%), and squamous cell carcinoma (SCC) or keratoacanthoma (KA; 20\%) [4]. Cases of granulomatous dermatitis, cutaneous T-cell lymphoma and pyogenic granuloma have also been reported in the literature [4, 5]. Dermoscopy has been used not only for early detection of skin cancers but also for identification of various skin lesions [6-11]. It is a useful method of skin examination to facilitate the decision which lesions need to be removed, allowing one to precisely determine the visible structures, and also to better understand the nature of adverse skin reactions.

\section{Aim}

The aim of our study was to describe the dermoscopic picture of different skin manifestations identified as skin toxicities during therapy with one of the BRAF inhibitors (vemurafenib). So far, only a few clinical reports on dermoscopic examination of skin lesions (mainly melanocytic lesions) occurring as skin toxicities with BRAF inhibitors are available in the PubMed database [12-15]. Therefore, we conducted clinical and dermoscopic examination of all skin lesions appearing in patients during therapy with vemurafenib.

\section{Material and methods}

Between October 2014 and June 2015, 8 patients ( 5 males and 3 females) with a median age of 53 years (range: 42-68 years), receiving vemurafenib therapy, were evaluated clinically and dermoscopically at the Department of Clinical and Experimental Oncology, Maria Sklodowska-Curie Memorial Cancer Center and Institute of Oncology in Gliwice. All patients had stage IV melanoma. Vemurafenib was given orally, and the initial dose was 960 mg twice a day. Skin toxicities were classified according to the Common Terminology Criteria for Adverse Events (CTCAE) version 4.3. The terms used for clinical manifestations of skin toxicities were based on definitions from the Mandala and Massi study [16]. All skin lesions occurring during therapy were examined using a hand-held dermoscope with polarised and nonpolarised light (DermLite DL3) at least once between the $2^{\text {nd }}$ and $15^{\text {th }}$ month of the therapy. Then they were stored in the videodermoscope Medicam 800 HD system and described. Skin lesions suspected of malignancy that appeared during therapy were totally surgically excised with consecutive histopathological examination. The patients were not receiving photosensitising drugs or immunosuppressants, and none of them had a history of any dermatological disorder. The study was approved by the local Ethics Committee in accordance with current regulations concerning human research (Approval No. KB/430-38/14).

\section{Results}

All examined patients developed skin toxicity. Detailed clinical and dermoscopic characteristics of the group of patients developing skin toxicities during BRAF inhibitor treatment are shown in Table 1.

The majority of patients (7/8) developed G1 skin toxicity according to CTCAE version 4.3. Only one had G2 acneiform rash and palmar-plantar erythrodysaesthesia. The most common adverse skin reaction was skin dryness (4/8 patients). Three patients had skin induration (3/8), photosensitivity (3/8), and palmar-plantar erythrodysaesthesia (3/8). Alopecia and pruritus occurred in 2 different patients. Only 1 patient demonstrated telangiectasia in a non-specific arrangement. Two patients had acneiform rash with comedones, in 1 case accompanied by intrafollicular plugs in dermoscopy (Figures 1 A-D). Whitish-yellowish, structureless steatotic cysts were seen dermoscopically in 2 patients (Figures $1 \mathrm{E}$, F). We also observed symmetric and asymmetric hyperkeratosis of the nipples in 5/8 patients; all these lesions showed in dermoscopy the presence of brownish-yellowish clods, which were sometimes angular-shaped (Figures $1 \mathrm{G}, \mathrm{H}$ ), and in 1 patient they were very confluent. One patient had keratosis pilaris with hyperkeratotic intrafollicular filiform plugs within normally visible dermoscopically pilosebaceous orifices (Figures 2 A, B). Palmar-plantar erythrodysaesthesia with a homogeneous yellowish pattern in dermoscopy occurred in 3 patients (Figures $2 \mathrm{C}$, D). Hyperkeratotic verrucas were found in 5 patients and dermoscopy revealed a structureless pattern in all examined lesions of this type. Moreover, central dots as well as exophytic proliferation were seen in 3 patients (Figures $2 \mathrm{E}, \mathrm{F}$ ), and hairpin vessels in 2 patients. Dermoscopy proved the presence of homogeneous haemorrhage in only 1 patient.

Whole-body dermoscopy revealed 2 suspected melanocytic lesions in 3 patients, and 1 non-melanocytic lesion. Dermoscopic indications for surgical excision of the suspected melanocytic lesions were the presence of an atypical pigmented network (abrupt cut-off, big holes) in 3 patients, as well as the occurrence of atypical globules and homogeneous blue pattern, which were seen in 2 patients. Surgical excision was recommended for all suspected skin lesions. Eventually, 2 patients consented to removal of their lesions. The other 2 patients did not accept the invasive nature of this procedure and did not consent to surgical excision. Histopathological results (2 lesions in 2 patients) confirmed their benign nature as a blue naevus (Figures $2 \mathrm{G}, \mathrm{H}$ ), compound naevus, acral compound naevus, and melanocytic naevus. 
Table 1. Detailed clinical and dermoscopic (DX) characteristics of the group of patients developing selected skin toxicities during vemurafenib therapy

\begin{tabular}{|c|c|c|c|c|c|c|c|c|}
\hline \multirow[t]{2}{*}{ Parameter } & \multicolumn{8}{|c|}{ Patients } \\
\hline & 1 & 2 & 3 & 4 & 5 & 6 & 7 & 8 \\
\hline Sex & M & M & M & $\mathrm{F}$ & $\mathrm{F}$ & M & M & $\mathrm{F}$ \\
\hline Age & 55 & 47 & 51 & 64 & 68 & 45 & 64 & 42 \\
\hline Month of dermoscopic assessment & 15 & 7 & 6 & 13 & 4 & 10 & 14 & 2 \\
\hline Skin dryness & & & G1 & & G1 & G1 & G1 & \\
\hline Skin induration & G1 & G1 & & & G1 & & & \\
\hline Alopecia & & & & G1 & & & G1 & \\
\hline Pruritus & & & G1 & & & & G1 & \\
\hline Photosensitivity & & G1 & G1 & & & & & G1 \\
\hline Telangiectasia & & & & & G1 & & & \\
\hline Palmar-plantar erythrodysaesthesia & G1 & & & & G1 & & & $\mathrm{G} 2$ \\
\hline DX of palmar-plantar erythrodysaesthesia - yellowish, structureless pattern & $x$ & & & & $\mathrm{x}$ & & & $x$ \\
\hline Acneiform rash & & & & & G1 & & & G2 \\
\hline DX of acneiform rash - comedones & & & & & $\mathrm{x}$ & & & $\mathrm{x}$ \\
\hline DX of acneiform rash - perifollicular plugs & & & & & & & & $x$ \\
\hline Steatotic cysts & & & & & $\mathrm{x}$ & $x$ & & \\
\hline DX of steatotic cyst - whitish-yellowish, structureless pattern & & & & & $\mathrm{x}$ & $x$ & & \\
\hline DX of keratosis pilaris - hyperkeratotic plug & & & & & & & $\mathrm{x}$ & \\
\hline Hyperkeratosis of the nipples - symmetric & & & $x$ & & $x$ & & $x$ & $x$ \\
\hline Hyperkeratosis of the nipples - asymmetric & $x$ & & & & & & & \\
\hline DX of nipple hyperkeratosis - brownish-yellowish clods & & & $x$ & & $\mathrm{x}$ & & $x$ & \\
\hline DX of nipple hyperkeratosis - brownish, yellowish angular clods & $x$ & & $\mathrm{x}$ & & $x$ & & $x$ & $x$ \\
\hline DX of nipple hyperkeratosis - confluent & & & & & & & $x$ & \\
\hline Hyperkeratotic verruca & & $x$ & $x$ & & $x$ & $x$ & $x$ & \\
\hline DX of verruca - structureless pattern & & $x$ & $x$ & & $x$ & $x$ & $x$ & \\
\hline DX of verruca - exophytic proliferation & & & & & $x$ & $\mathrm{x}$ & $x$ & \\
\hline DX of verruca - central dots & & $x$ & $x$ & & $x$ & & & \\
\hline DX of verruca - hair-pin vessels & & $x$ & & & $x$ & & & \\
\hline DX of verruca - haemorrhages & & & & & $x$ & & & \\
\hline Number of suspected melanocytic lesions & 2 & 2 & & 1 & & 2 & & \\
\hline DX of suspected melanocytic lesions - radial streaks & $x$ & $x$ & & & & & & \\
\hline DX of suspected melanocytic lesions - pseudopods & $x$ & $x$ & & & & & & \\
\hline DX of suspected melanocytic lesions - atypical network (abrupt cut-off, big holes) & $x$ & $x$ & & & & $\mathrm{x}$ & & \\
\hline DX of suspected melanocytic lesions - atypical globules & $x$ & & & & & $x$ & & \\
\hline DX of suspected melanocytic lesions - blue, homogeneous pattern & & & & $x$ & & $x$ & & \\
\hline Number of suspected keratoacanthomas & & & & 1 & & & & \\
\hline $\begin{array}{l}\text { DX of suspected keratoacanthoma - central hyperkeratotic plug, prominent linear } \\
\text { and serpentine vessels }\end{array}$ & & & & $\mathrm{x}$ & & & & \\
\hline
\end{tabular}



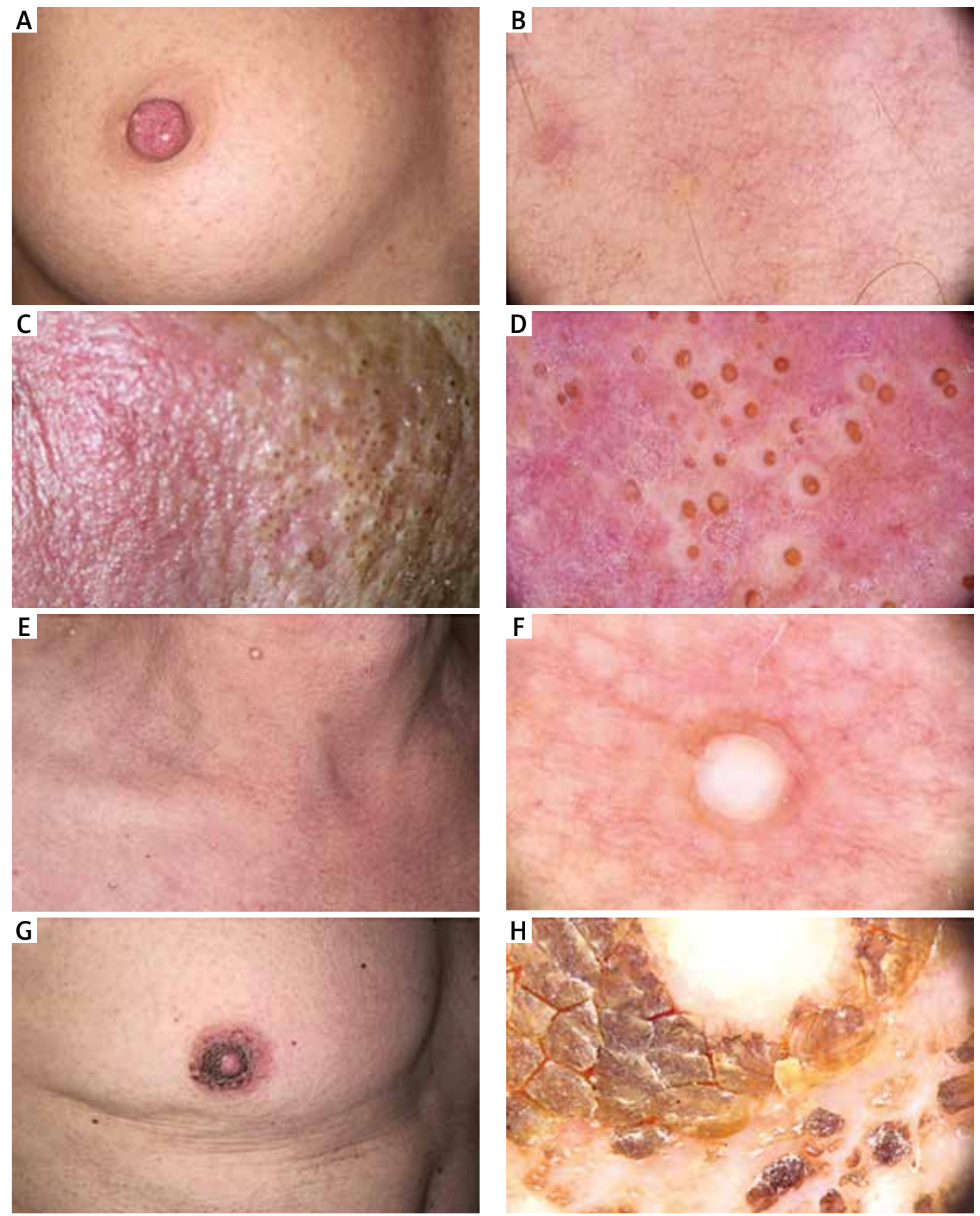

Figure 1. Clinical and dermoscopic images of patients developing skin toxicities during vemurafenib therapy. Clinical manifestation of G2 disseminated acneiform rash developing on the extremities, thorax, décolleté and abdominal region in patient 8 (A). Dermoscopy of acneiform rash demonstrated intrafollicular yellowish plugs, surrounded by multiple, wellvisible erythralgic telangiectasia in patient 8 (B). Clinical symptoms of acneiform rash consisted of black, multiple megacomedones with erythema of the face in patient 5 (C). Dermoscopy showed multiple, black-headed open megacomedones, grouped in clusters, localised on a reddish background as erythema resulting from phototoxicity in patient 5 (D). Clinical picture of yellowish, multiple steatotic cysts localised all over the upper trunk and neck in patient 5 (E). Dermoscopy of steatotic cysts showed yellowish and whitish, multiple, well-defined, rounded structures corresponding with its clinical picture in patient $5(\mathbf{F})$. Clinical picture of aggravated hyperkeratosis of the nipples suggesting "neglected nipples" in patient $1(\mathbf{G})$. Dermoscopy of hyperkeratosis of the nipples revealed the presence of multiple, angulated, hyperkeratotic clods, whitish to yellowish, resembling "dirty skin" in patient $1(\mathrm{H})$ 

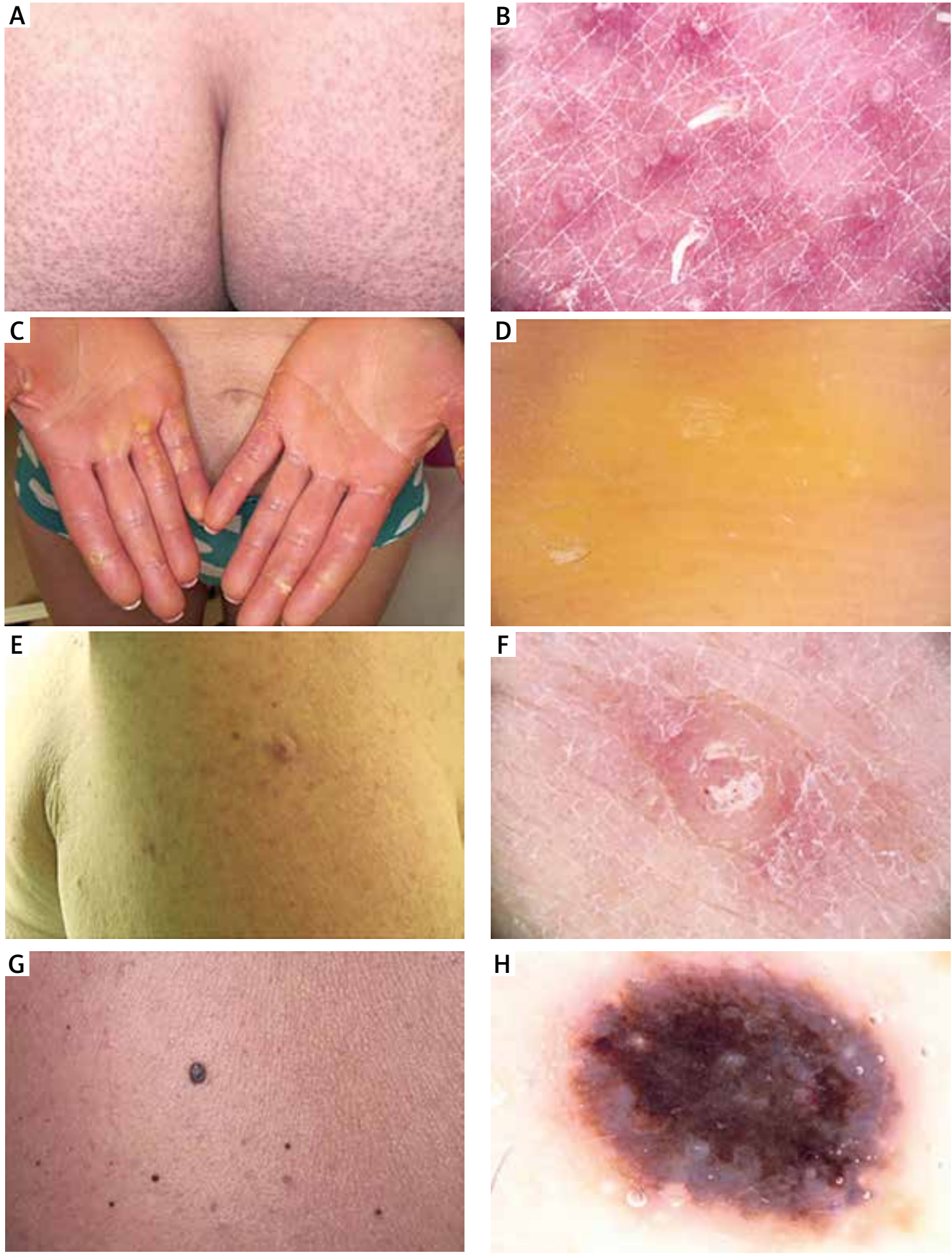

Figure 2. Clinical and dermoscopic images of patients developing skin toxicities during vemurafenib therapy. Clinical picture of exacerbated keratosis pilaris in patient 7 (A). Dermoscopy revealed multiple hyperkeratotic intrafollicular filiform plugs within normal visible pilosebaceous orifice (B). Clinical picture of palmar-plantar erythrodysaesthesia (G2) in patient 8 (C). Dermoscopy revealed hyperkeratotic skin changes in the form of yellowish, confluent, homogeneous masses in patient 8 (D). Clinical picture of proliferative, hyperkeratotic verruca in patient 5 (E). Dermoscopy showed several dotted vessels in an exophytic, amorphous proliferation (F). Clinical picture of suspected melanocytic lesion appeared as an "ugly duckling" lesion in patient 6 (G). Dermoscopically it was a lesion with a confluent, bluish-blackish homogeneous pattern suggesting melanoma, histopathologically identified as a blue naevus in patient $6(\mathbf{H})$ 
We found only 1 non-melanocytic lesion, which was suspected to be keratoacanthoma. Dermoscopy revealed a central hyperkeratotic plug with prominent linear and serpentine vessels highly suggestive for keratoacanthoma. Despite our recommendation, the patient did not agree to a surgical procedure, so histopathological diagnosis was not possible.

\section{Discussion}

Despite many reports in the literature on skin toxicity caused by BRAF inhibitors during treatment of melanoma, dermoscopic assessment of skin toxicities has rarely been described in the PubMed database [12-18]. One of the most frequent dermatoscopic findings in our study was hyperkeratosis of the nipples, with was seen quite commonly in our patients (5/8) as diffuse, hyperkeratotic, yellowish to brown papules coalescing to plaques within the nipples, correlated with the previously described dermoscopic pictures presenting yellowish to brown, angulated, keratotic clods in "neglected nipples" [9]. As we know from the Mandala et al. study [19], this author describes hyperkeratosis of the nipples and palmar regions. Keratosis pilaris was characterised dermoscopically by the presence of hyperkeratotic, filiform masses arising from normal follicular orifices. In this case, we did not observe the previously described widening of the follicular orifice or circular hair shafts [20, 21]. Palmar-plantar erythrodysaesthesia was characterised dermoscopically by a homogeneous, yellowish pattern typical for keratinisation disorders [22], corresponding with its clinical manifestation as yellowish, hyperkeratotic plaques localised on the pressure points on the sole of the foot and the palm [23].

In our study, we observed hyperkeratotic verrucas in 5 of 8 patients; also Boussemart et al. [23] reported this sign as very common - it was found in $79 \%$ of their patients [23].

Dermoscopic pictures of hyperkeratotic verrucas corresponded with previously described dermoscopic features including exophytic proliferation, dotted and hairpin vessels, as well as homogeneous haemorrhage [22, $24,25]$. According to the literature, dermoscopic examination of BRAF inhibitor-induced acantholytic dyskeratosis shows a pattern with a central branched polygonal brownish area surrounded by a thin whitish halo [18].

Another sign, rash, was present in 2 of 8 patients. This rate was lower compared to the Sinha et al. [4] and Boussemart et al. studies (64\% and 55\%, respectively) [23].

In our study, the dermoscopic pattern of acneiform rash with comedones showed the presence of intrafollicular yellowish plugs, occasionally surrounded by small telangiectasia, in more severe cases forming widened intrafollicular plugs filled by brownish masses of keratin. Dermoscopy of typical comedones in acne vulgaris may indicate the presence of numerous, homogeneous areas, light or dark brown (at times black) in colour, depending on the type of acne (open or closed comedones), predominantly circular [26]. Whitish-yellowish steatotic cysts revealed a structureless dermoscopic pattern [27].

New eruptive melanocytic naevi $[19,28]$, dysplastic naevi and new primary melanomas arising during BRAF inhibitor therapy have been most commonly described in the literature [16]. In the Goppner et al. study [16], 4 of 13 (31\%) patients developed 4 new naevi-associated malignant melanomas. All melanocytic tumours were associated with pre-existing naevi that had clinically changed over a period of 6 weeks to 6 months of therapy with vemurafenib, and showed malignant dermoscopic changes, such as an irregular, darkened or distorted pigmentation network and prominent unilateral globules and dots, mainly in the centre of the tumour. In 4 of our patients we also found similar dermoscopic features suggesting the highly suspicious nature of these lesions. The changes within the naevi seen in dermoscopy during anti-BRAF therapy predominantly involved involution, a decrease in pigmentation and size, as well as flattening of raised naevi [29]. In the study of McClenahan et al. [29] 71\% of unspecific and $26 \%$ of reticular naevi showed signs of involution, while both raised globular naevi decreased in pigmentation and flattened [29], which was also reported in a previously published study [30]. Haenssle et al. [30] highlighted three types of naevus changes seen in dermoscopy during anti-BRAF therapy: (1) involuted naevi, originally with centrally elevated papillomatous and a predominant globular pattern; (2) pre-existing nevi that increased in size and started showing atypical pigmentation (such lesions were flat and showed a predominant reticular pattern at baseline); (3) multiple new naevi [30].

It is necessary to mention the importance of confocal microscopy in the diagnosis and monitoring of skin lesions; however, digital dermoscopy remains an important, useful diagnostic tool and it has been widely used both in daily practice and in clinical trials [31, 32].

Our study had a significant limitation - all patients were dermoscopically examined only once during therapy. We realise that skin lesion monitoring over time can improve the diagnostic value of dermoscopy [17, 33]. Ferrara et al. [33] demonstrated that dermoscopy can also be used to assess the potential progression of metastatic melanoma of the skin treated with a BRAF inhibitor [33]. We decided to excise 4 suspected lesions. However, none of them proved to be malignant. Even the most atypical lesion that occurs during vemurafenib therapy may be benign in histopathological examination [30].

In our study, the excised lesions were not tested for BRAF mutation. However, recent clinical trials have focused on the dermoscopic features of lesions depending of the BRAF mutation status [34]. Bombonato et al. [34] found in their study that ulceration and irregular peripheral streaks represented dermoscopic features indicative for BRAF-mutated melanoma, while dotted vessels were 
suggestive for wild-type melanoma [34]. Dermoscopic features of BRAF V600K-mutated melanoma included reticular grey-blue areas and white regression; each of these signs was present in $50 \%$ of cases analysed by Ponti et al. [35].

It seems that the dynamics of each skin lesion and their dermoscopic features partially depend on the BRAF mutation status $[29,35,36]$. Dermoscopically defined globular naevi, which are most often composed of large melanocytic nests, and lesions with a predominantly dermal growth pattern, are at least three times more likely to express BRAF V600E than dermoscopically defined reticular naevi [36].

Dermoscopy may also be used for monitoring regression of metastatic melanoma lesions during antiBRAF therapy [17]. Dermoscopy of treated lesions revealed thepresence of a bluish colour. However instead of structures, as in blue-naevus-like or other common metastatic patterns, there are regressive features that typically correspond to inflammation and fibrosis. Histopathology examination confirms, that regressive features typified all pigmented areas were negative for the presence of melanoma metastatic tumor [17].

Despite the above-mentioned limitations of our study, we demonstrated that if for various reasons skin dermoscopy monitoring is not feasible, even a single dermoscopic examination during vemurafenib therapy can be very helpful in assessing skin lesions.

Dermoscopic images are specific for selected skin toxicities resulting from therapy with a BRAF inhibitor, correlating with their clinical manifestations. In patients who should be monitored for melanocytic proliferations, skin cancers and developing skin toxicities, dermoscopy remains a useful, easy-to-perform diagnostic method for visualisation and identification of many dermatological lesions.

\section{Acknowledgments}

Two first authors contributed equally to this work.

\section{Conflict of interest}

The authors declare no conflict of interest.

\section{References}

1. Garbe C, Peris K, Hauschild A et al. Diagnosis and treatment of melanoma. European consensus-based interdisciplinary guideline - update 2016. Eur J Cancer 2016; 63: 201-17.

2. Sullivan RJ, Flaherty KT. New strategies in melanoma: entering the era of combinatorial therapy. Clin Cancer Res 2015; 21: 2424-35.

3. McArthur GA, Chapman PB, Robert C, et al. Safety and efficacy of vemurafenib in BRAF(V600E) and BRAF(V600K) mutation-positive melanoma (BRIM-3): extended follow-up of a phase 3, randomised, open-label study. Lancet Oncol 2014; 15: 323-32.
4. Sinha R, Larkin J, Gore M, Fearfield L. Cutaneous toxicities associated with vemurafenib therapy in 107 patients with BRAF V600E mutation-positive metastatic melanoma, including recognition and management of rare presentations. Br J Dermatol 2015; 173: 1024-31.

5. Henning B, Stieger P, Kamarachev J, et al. Pyogenic granuloma in patients treated with selective BRAF inhibitors: another manifestation of paradoxical pathway activation. Melanoma Res 2016; 26: 304-7.

6. Zalaudek I, Lallas A, Moscarella E, et al. The dermatologist's stethoscope - traditional and new applications of dermoscopy. Dermatol Pract Concept 2013; 3: 67-71.

7. Kaminska-Winciorek G, Giebel S, Lange D, Maciejewski A. Dermoscopy in near-full facial transplantation. J Am Acad Dermatol 2015; 72 (1 Suppl.): S19-21.

8. Kaminska-Winciorek G, Wydmanski J, Scope A, et al. "Neglected nipples": acanthosis nigricans-like plaques caused by avoidance of nipple cleansing. Dermatol Pract Concept 2014; 4: 81-4.

9. Kaminska-Winciorek G, Wydmanski J. Dermoscopy of skin metastases from breast cancer and of the orange peel type ("peau d'orange"): a report of two cases. Int J Dermatol 2015; 54: 343-6.

10. Kaminska-Winciorek G. Dermoscopy of nevus spilus. Dermatol Surg 2013; 39: 1550-4.

11. Kaminska-Winciorek G, Wydmanski J, Januszewski K, Silny W. Dermoscopy of nodular skin metastases from the gastrointestinal primary cancer. Postep Dermatol Alergol 2015; 32: 312-6.

12. Anforth RM, Carlos GR, Scolyer RA, et al. Eruptive naevi in a patient treated with LGX818 for BRAF mutant metastatic melanoma. Melanoma Res 2015; 25: 91-4.

13. Gerami P, Sorrell J, Martini M. Dermatoscopic evolution of dysplastic nevi showing high-grade dysplasia in a metastatic melanoma patient on vemurafenib. J Am Acad Dermatol 2012; 67: 275-6.

14. Haenssle HA, Kraus SL, Brehmer F, et al. Dynamic changes in nevi of a patient with melanoma treated with vemurafenib: importance of sequential dermoscopy. Arch Dermatol 2012; 148: 1183-5.

15. Perier-Muzet M, Thomas L, Poulalhon N, et al. Melanoma patients under vemurafenib: prospective follow-up of melanocytic lesions by digital dermoscopy. J Invest Dermatol 2014; 134: 1351-8.

16. Goppner D, Muller J, Kruger S, et al. High incidence of naeviassociated BRAF wild-type melanoma and dysplastic naevi under treatment with the class I BRAF inhibitor vemurafenib. Acta Derm Venereol 2014; 94: 517-20.

17. Ribero S, Marra E, Tomasini CF, et al. Confocal microscopy and dermoscopy for the monitoring of BRAF inhibitor therapy of melanoma skin metastases. Br J Dermatol 2017; 176: 1101-2.

18. Errichetti E, Stinco G. Dermoscopy in general dermatology: a practical overview. Dermatol Ther (Heidelb) 2016; 6: 471507.

19. Mandala M, Massi D, De Giorgi V. Cutaneous toxicities of BRAF inhibitors: clinical and pathological challenges and call to action. Crit Rev Oncol Hematol 2013; 88: 318-7.

20. Silverberg NB. A pilot trial of dermoscopy as a rapid assessment tool in pediatric dermatoses. Cutis 2011; 87: 148-54.

21. Thomas M, Khopkar US. Keratosis pilaris revisited: is it more than just a follicular keratosis? Int J Trichology 2012; 4: 255-8. 
22. Bae JM, Kang H, Kim HO, Park YM. Differential diagnosis of plantar wart from corn, callus and healed wart with the aid of dermoscopy. Br J Dermatol 2009; 160: 220-2.

23. Boussemart L, Routier E, Mateus C, et al. Prospective study of cutaneous side-effects associated with the BRAF inhibitor vemurafenib: a study of 42 patients. Ann Oncol 2013; 24: 1691-7.

24. Lee DY, Park JH, Lee KH, et al. The use of dermoscopy for the diagnosis of plantar wart. J Eur Acad Dermatol Venereol 2009; 23: 726-7.

25. Zalaudek I, Giacomel J, Cabo H, et al. Entodermoscopy: a new tool for diagnosing skin infections and infestations. Dermatology 2008; 216: 14-23.

26. Kamińska-Winciorek G, Spiewak R. Dermoscopy on nevus comedonicus: a case report and review of the literature. Postep Dermatol Alergol 2013; 30: 252-4.

27. Alfaro-Castellón P, Mejía-Rodríguez SA, Valencia-Herrera A, et al. Dermoscopy distinction of eruptive vellus hair cysts with molluscum contagiosum and acne lesions. Pediatr Dermatol 2012; 29: 772-3.

28. Ankudowicz A, Brzezicka-Ciach U, Wenska B. Cutaneous side-effects during therapy of melanoma by vemurafenib. Przegl Dermatol 2015; 102: 221-6.

29. McClenahan P, Lin LL, Tan JM, et al. BRAFV600E mutation status of involuting and stable nevi in dabrafenib therapy with or without trametinib. JAMA Dermatol 2014; 150: 107982.

30. Haenssle HA, Kraus SL, Brehmer F, et al. Dynamic changes in nevi of a patient with melanoma treated with vemurafenib: importance of sequential dermoscopy. Arch Dermatol 2012; 148: 1183-5.

31. Ruini C, Manfredini M, Pellacani G, et al. Confocal microscopy characterization of BRAFV600E mutated melanomas. Melanoma Res 2015; 25: 367-71.

32. Debarbieux S, Dalle S, Depaepe L, et al. Second primary melanomas treated with BRAF blockers study by reflectance confocal microscopy. Br J Dermatol 2013; 168: 1230-5.

33. Ferrara G, Cacitti V, Improta G, et al. Epidermotropic progression of melanoma during therapy with vemurafenib and trametinib. J Am Acad Dermatol 2016; 75: e133-5.

34. Bombonato C, Ribero S, Pozzobon FC, et al. Association between dermoscopic and reflectance confocal microscopy features of cutaneous melanoma with BRAF mutational status. J Eur Acad Dermatol Venereol 2017; 31: 643-9.

35. Ponti G, Manfredini M, Tomasi A, Pellacani G. Distinctive clinical and dermoscopic features of BRAF V600K mutated melanomas. Br J Dermatol 2015; 172: 1438-40.

36. Marchetti MA, Kiuru MH, Busam KJ, et al. Melanocytic naevi with globular and reticular dermoscopic patterns display distinct BRAF V600E expression profiles and histopathological patterns. Br J Dermatol 2014; 171: 1060-5. 